\title{
The Discovery of Raman Lines in NGC 7027
}

\author{
D. Péquignot ${ }^{1}$, J.-P. Baluteau ${ }^{2}$, C. Boisson ${ }^{1}$ and C. Morisset ${ }^{1}$ \\ ${ }^{1}$ Observatoire de Meudon, F-92195 Meudon Cédex, France; \\ ${ }^{2}$ Laboratoire d'Astronomie Spatiale, BP 8, F-13376 Marseille Cédex 12, France
}

Broad emission lines $\lambda \lambda 682.5,708.2 \mathrm{~nm}$, observed for years in the spectrum of symbiotic stars, have been identified by Schmid (1989) as due to Raman scattering of O VI 103.2, $103.6 \mathrm{~nm}$ by $\mathrm{H}^{0}$. In one symbiotic star, van Groningen (1993) detected Raman scattered He II.

Raman scattering may be regarded as a two-photon process: it is the inelastic counterpart to Rayleigh scattering. Raman scattering is well known for molecules but is also relevant to atomic hydrogen. In this process a photon is usually captured from $\mathrm{H}^{0}(1 s)$ into an intermediate (continuum) state which promptly decays onto an $n s$ or $n d$ state. The Raman-scattering cross section strongly peaks at the resonance energies corresponding to the Lyman lines (Isliker et al., 1989). If the final state is $2 s$, the scattered photon will appear in the optical range.

If an $\mathrm{H}^{0}$ region with sufficient column density is present in a planetary nebula and if a spectral feature arising from either the central star or the $\mathrm{H}^{+}$region happens to nearly coincide with a Lyman line, a Raman feature will appear in the vicinity of one of the Balmer lines.

Optical Raman lines will be 5 to 6 times broader than normal lines, providing accurate diagnostics of velocity fields in nebulae. Also the Raman-conversion efficiency and the degree of polarization depend on the mass and the distribution of the gas (e.g., Schild \& Schmid, 1996 and references therein).

Here, we report on the discovery of three He II Raman lines at wavelengths $\lambda 654.5$, $\lambda 485.1$, and $\lambda 433.2 \mathrm{~nm}$ in the spectrum of the planetary nebula NGC 7027. Raman lines are identified for the first time in a planetary nebula (Péquignot et al., 1996). The former line has not been reported before in any astronomical object (Péquignot et al., 1997). Other Raman features are suspected in the wings of the Balmer lines.

Raman scattering originates in the photodissociation region (PDR) of NGC 7027, at the interface between the $\mathrm{H}^{+}$and $\mathrm{H}_{2}$ regions. The mass of the PDR is about $0.5 \mathrm{M}_{\odot}$ and its expansion velocity is $17 \mathrm{~km} \mathrm{~s}^{-1}$, intermediate between those of the $\mathrm{H}^{+}$and $\mathrm{H}_{2}$ regions. The efficiency of He II Raman conversion is larger in NGC 7027 than in symbiotic stars, presumably because the covering factor of the $\mathrm{H}^{+}$region by the $\mathrm{H}^{0}$ region is larger.

Raman scattering by hydrogen is pointed out as a new powerful tool to detect and study atomic hydrogen in planetary nebulae and other nebulae using deep optical spectroscopy.

\section{REFERENCES}

Isliker, H., Nussbaumer, H., Vogel, M., 1989, Astron. Astrophys., 219, 271

Péquignot, D., Baluteau, J.-P., Morisset, C., Boisson, C., 1996, Astron. Astrophys., submitted Péquignot, D., Morisset, C., Baluteau, J.-P., 1997, Astron. Astrophys., in preparation

Schmid, H.M., 1989, Astron. Astrophys. Lett., 211, L31

Schild, H., Schmid, H.M., 1996, Astron. Astrophys., 310, 211

van Groningen, E., 1993, Monthly Not. Roy. Astron. Soc., 264, 975 